\title{
Association entre le travail par quarts et l'obésité chez les hommes dans le nord-est de l'Ontario
}

\author{
Anne Grundy, Ph. D. (1,2); Michelle Cotterchio, Ph. D. (3,4); Victoria A. Kirsh, Ph. D. (4); Victoria Nadalin, M.A. (3); \\ Nancy Lightfoot, Ph. D. (5); Nancy Kreiger, M.S.P., Ph. D. (4,6)
}

Cet article a fait l'objet d'une évaluation par les pairs.

Diffuser cet article sur Twitter

\section{Résumé}

Introduction : Même si certaines études suggèrent une association entre le travail par quarts et l'obésité, peu sont fondées sur la population ou tiennent compte de divers horaires de travail par quarts. L’obésité étant associée à plusieurs problèmes de santé chroniques, il est important de comprendre quelles formes de travail par quarts ont une incidence sur elle et d'effectuer des travaux permettant d'évaluer de façon plus détaillée l'exposition au travail par quarts.

Méthodologie : Au moyen d'une régression logistique polytomique multivariée, nous avons étudié l'association entre le travail par quarts (de soir ou nuit, par quarts ou autre type de rotation) et le surpoids et l'obésité, en fonction d'une mesure transversale de l'indice de masse corporelle chez 1561 hommes. Ces hommes avaient déjà servi de témoins dans une étude cas-témoins sur le cancer de la prostate menée dans le nord-est de l'Ontario de 1995 à 1999. Nous avons obtenu l'information sur leurs antécédents de travail (notamment sur le travail par quarts), leur taille et leur poids à partir de données autodéclarées recueillies par questionnaire.

Résultats : Nous avons observé une association entre le fait d'avoir déjà travaillé par quarts (par opposition au fait de n'avoir jamais travaillé par quarts) et le surpoids (RC [rapport de cotes] $=1,34$; IC [intervalle de confiance] à $95 \%: 1,05$ à 1,73) ainsi que l'obésité $(\mathrm{RC}=1,57$; IC à $95 \%: 1,12$ à 2,21). Nous avons également observé des associations statistiquement non significatives avec le fait d'avoir déjà travaillé (par opposition au fait de n'avoir jamais travaillé) de façon permanente selon un quart de soir ou de nuit. Nous avons par ailleurs observé une tendance à la hausse statistiquement significative en ce qui concerne le risque de surpoids et d'obésité en fonction de la durée du travail par quarts.

Conclusion : Tant l'association positive observée entre le travail par quarts et l'obésité que l'association positive suggérée dans notre étude en ce qui concerne le travail permanent selon un quart de soir ou nuit concordent avec ce qui a été observé antérieurement. D'autres études en population tenant compte de nos résultats seront à mener pour examiner d'autres caractéristiques du travail par quarts, afin de mieux déterminer si certains types de travail par quarts ont une plus grande incidence sur l'obésité que d'autres.

Mots-clés : travail par quarts, obésité, hommes, recherche en population

\section{Introduction}

Le travail par quarts est considéré comme un facteur de risque pour de nombreux problèmes de santé chroniques dans lesquels l'obésité joue un rôle $\mathrm{e}^{1-6}$. Plus précisément, on a observé que les risques de maladie cardiovasculaire ${ }^{1}$, de diabète de type $2^{2,3}$, de syndrome métabolique ${ }^{4,5}$ et de

\section{Points saillants}

- Nous avons examiné l'association entre le fait d'avoir occupé un emploi incluant du travail de nuit (soir ou nuit, par quarts ou autre type de rotation) et le surpoids et l'obésité chez des hommes du nord-est de l'Ontario.

- Les hommes ayant déjà travaillé par quarts étaient plus susceptibles d'accuser un surpoids ou d'être obèses que ceux n'ayant jamais travaillé par quarts, et une observation similaire a été faite pour les hommes ayant déjà travaillé de soir ou de nuit.

- Nous avons par ailleurs observé une tendance à la hausse statistiquement significative en ce qui concerne le risque de surpoids et d'obésité en fonction de la durée du travail par quarts.

- Étant donné que l'obésité est associée à plusieurs problèmes de santé chroniques, il est important de comprendre quels types de travail par quarts sont associés à l'obésité, afin de mettre au point les politiques relatives au travail les plus favorables possible du point de vue de la santé des travailleurs.

cancer $^{6}$ sont plus importants chez les personnes qui travaillent par quarts.

Des études transversales ${ }^{4,7-17}$, de cohorte ${ }^{18-23}$ et longitudinales ${ }^{24-26}$ ont été menées pour évaluer le lien entre le travail par quarts et l'obésité. Certains chercheurs ont constaté

Rattachement des auteures :

1. Centre de recherche du Centre hospitalier de l'Université de Montréal (CRCHUM), Montréal (Québec), Canada

2. Département de médecine sociale et préventive, Université de Montréal, Montréal (Québec), Canada

3. Prévention et lutte contre le cancer, Action Cancer Ontario, Toronto (Ontario), Canada

4. École de santé publique Dalla Lana, Université de Toronto, Toronto (Ontario), Canada

5. École de santé dans les milieux ruraux et du Nord, Université Laurentienne, Sudbury (Ontario), Canada

6. Département des sciences de la nutrition, Université de Toronto, Toronto (Ontario), Canada

Correspondance : Anne Grundy, Centre de recherche du Centre hospitalier de l'Université de Montréal, 850, rue Saint-Denis, 2e étage, Montréal (Québec) H2X 0A9; tél. : 514-890-8000, poste 15921; courriel : anne.grundy@umontreal.ca 


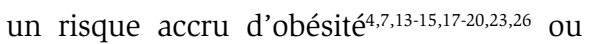
de prise pondérale ${ }^{9}$ chez les travailleurs de quarts, tandis que d'autres n'ont observé aucune association ${ }^{8-12,16,24,25}$ de ce type, et ces résultats ne semblent pas dépendre du plan d'étude. La plupart des études antérieures sur le travail par quarts et l'obésité ont été menées au sein de certains types de milieux de travail, ont été généralement limitées à des personnes travaillant au sein d'une même industrie $7,8,10-15,17,19,20,23,24,26,27$ et peu d'entre elles étaient basées sur la population, en particulier chez les hommes $^{4,18}$. Par conséquent, l'évaluation des effets du travail par quarts demeure limitée, d'autant plus que la plupart des études portent sur un type bien précis d'horaire ou de travail par quarts , $8-17,19-28$.

De plus, bien que la plupart des études aient utilisé des travailleurs de jour seulement comme groupe de référence $e^{4,7-15,17,19-23,25,27}$, certaines ne comparaient que les effets des différents horaires de travail par quarts sans recourir à un groupe réellement " non exposé $»^{16,24,26}$. Il s'est donc révélé nécessaire de mener des études permettant d'évaluer le travail par quarts de façon plus détaillée (p. ex. par type de quart et en fonction du nombre cumulatif d'années passées à travailler par quarts) ${ }^{28}$. Notre étude en population, qui porte sur plus de 1500 hommes du nord-est de l'Ontario (Canada), va plus loin que les travaux antérieurs, et ce, à plusieurs titres. En particulier, elle offre une évaluation détaillée du travail par quarts qui touche des emplois au sein de multiples industries, selon des horaires par quarts en rotation et permanents, ce qui permet d'examiner la relation entre les antécédents en matière de travail par quarts et l'obésité chez les hommes.

\section{Méthodologie}

\section{Échantillon de l'étude}

Nous avons analysé les données fournies par les personnes de référence (témoins) ayant participé à une étude cas-témoins sur le cancer de la prostate menée dans le nord-est de l'Ontario de 1995 à 199929,30, qui a porté sur l'association entre des facteurs professionnels et non professionnels et le risque de cancer de la prostate. Ces personnes de référence étaient des hommes de 45 à 84 ans n'ayant aucun antécédent de cancer de la prostate et choisis aléatoirement au moyen d'une liste de numéros de téléphone résidentiels du nord-est de l'Ontario ${ }^{29,30}$. Les données ayant servi de source à notre analyse sont tirées des réponses fournies par ces personnes à un questionnaire envoyé par la poste qui visait à recueillir de l'information sur un certain nombre de caractéristiques personnelles. Comme nous l'avons mentionné précédemment, le taux de réponse des témoins dans cette étude initiale était de $47,5 \%{ }^{29}$, avec à disposition au total pour notre analyse les données de 1622 hommes. L'approbation du volet éthique de l'étude initiale a été fournie par le comité d'éthique en matière de recherche de l'Hôpital Laurentien, et l'approbation pour notre analyse a été fournie par le comité d'éthique de l'Institut de recherche Horizon Santé-Nord et le comité d'éthique en matière de recherche de l'Université Laurentienne.

\section{Évaluation du travail par quarts}

Un volet sur les antécédents professionnels a été intégré au questionnaire de l'étude initiale. On demandait aux hommes d'indiquer tous les emplois qu'ils avaient occupés au cours de leur vie. Les participants devaient indiquer l'âge qu'ils avaient au début et à la fin de chaque emploi afin qu'on puisse calculer les durées d'emploi, et ils devaient indiquer le type d'horaire selon lequel ils travaillaient (de jour seulement, quart de soir ou nuit, par quarts en rotation ou autre), afin qu'il soit possible de caractériser leurs antécédents en matière de travail par quarts. La notion de " travail par quarts " a été définie comme étant le fait d'avoir travaillé individuellement selon un quart de soir ou nuit, par quarts en rotation ou selon une autre formule (" personnes ayant déjà travaillé par quarts »). Nous avons estimé la durée à vie du travail par quarts en additionnant le nombre d'années passées à travailler par quarts pour l'ensemble des emplois et en divisant la durée par deux dans le cas des emplois à temps partiel.

\section{Évaluation de l'obésité}

L’obésité a été évaluée au moyen de l'indice de masse corporelle (IMC, en $\mathrm{kg} / \mathrm{m}^{2}$ ). $\mathrm{Au}$ moment de remplir le questionnaire, les participants devaient indiquer leur taille et leur poids cinq ans plus tôt ainsi que leur taille au début de la trentaine et leur poids au début de la trentaine et de la cinquantaine. L'IMC associé aux cinq années précédant la participation à l'étude a été estimé, car dans le cadre de l'étude cas-témoins initiale sur le cancer de la prostate ${ }^{29}$ on s'intéressait à l'IMC avant l'apparition du cancer chez les participants atteints du cancer de la prostate. Afin de nous assurer que les données associées aux personnes de référence étaient comparables aux données associées aux patients atteints du cancer de la prostate, nous avions également évalué l'IMC de l'ensemble des personnes de référence cinq ans avant leur participation à l'étude et nous avions utilisé ces personnes de référence pour l'analyse. Ainsi, l'IMC utilisé ici pour caractériser l'obésité est associé aux cinq années précédant la participation à l'étude. Les hommes ayant un IMC inférieur à 25 ont été considérés comme ayant un poids normal, ceux ayant un IMC de 25 à moins de 30 ont été considérés comme étant en surpoids et ceux ayant un IMC égal ou supérieur à 30 ont été considérés comme obèses ${ }^{31}$.

Afin de réduire les données manquantes au sujet de l'IMC, nous avons utilisé la taille des participants au début de la trentaine lorsqu'elle était disponible et que nous ne disposions pas de leur taille cinq ans avant l'étude. De même, lorsque nous ne disposions pas du poids des participants cinq ans avant l'étude, nous avons utilisé pour les hommes de 50 à 59 ans leur poids au début de la cinquantaine. (Les hommes des autres groupes d'âge dont le poids n'était pas disponible ont été exclus, car il n'y avait aucune donnée de substitution acceptable. Les hommes devaient avoir au moins 45 ans pour participer à l'étude, de sorte que leur poids au début de la trentaine n'était pas une variable de substitution acceptable pour la période de cinq ans précédant l'étude.) Cette méthode alternative du calcul de l'IMC a été utilisée pour 51 (11 \%) participants de poids normal, 104 (12\%) participants en surpoids et 34 (14 \%) participants obèses. Nous ne dispositions d'aucune donnée sur la taille ou le poids pour 61 hommes, ce qui a rendu impossible la détermination de leur IMC.

\section{Évaluation des variables de confusion potentielles}

Les variables de confusion potentielles en ce qui concerne la relation entre le travail par quarts et l'obésité étaient l'âge, l'état matrimonial, le statut socio-économique, l'activité physique, la consommation énergétique totale (alimentation) et le tabagisme $^{28}$. Nous avons utilisé le niveau de scolarité et le revenu familial pour caractériser le statut socio-économique, et nous 
avons utilisé l'activité générale ainsi que l'activité associée au travail pour caractériser l'activité physique. En ce qui concerne l'activité physique générale, les hommes devaient déclarer le nombre de fois par mois où ils faisaient une activité physique intense ou modérée durant au moins 20 minutes, et ce, pendant quatre périodes de vie (l'adolescence, la trentaine, la cinquantaine et cinq ans avant l'étude). Les données sur l'activité physique intense et l'activité physique modérée ont été recueillies au moyen de questions distinctes, assorties d'exemples de chaque type d'activité. Nous avons créé un indice d'activité physique en additionnant le nombre de fois où les hommes avaient dit effectuer des activités physiques modérées ou intenses pendant chacune des quatre périodes précitées, puis nous avons créé des quartiles de l'indice d'activité physique, où les valeurs élevées représentent les personnes les plus actives au cours de leur vie.

Pour l'évaluation de l'activité physique associée au travail, les hommes devaient classer les activités habituelles effectuées dans le cadre de leur emploi en fonction de quatre catégories (travail en position assise ou activité légère, modérée ou intense), pendant quatre périodes de vie (début de la vingtaine, début de la trentaine, début de la cinquantaine et cinq ans avant l'étude). Nous avons catégorisé l'activité physique associée au travail en fonction du nombre de périodes au cours desquelles les hommes avaient indiqué effectuer des activités modérées ou intenses dans le cadre de leur emploi $(0,1,2,3$ ou 4 périodes). Nous avons évalué leur alimentation deux ans avant leur participation à l'étude en utilisant les données associées aux antécédents alimentaires complets recueillies au moyen du questionnaire, et à partir desquelles nous avions estimé leur consommation énergétique totale dans une analyse antérieure ${ }^{30}$. Cette dernière a été mesurée de façon continue et en quartiles. Quant au tabagisme, il a été défini comme le fait d'avoir déjà fumé des cigarettes avec ou sans filtre, et nous avons réparti les hommes en trois catégories (personnes n'ayant jamais fumé, ex-fumeurs, fumeurs).

\section{Analyse statistique}

Nous avons décrit les caractéristiques des hommes classés comme ayant un poids normal, en surpoids ou obèses en utilisant des moyennes et des écarts types pour les variables continues et des pourcentages pour les variables nominales. Nous avons également stratifié les caractéristiques descriptives des participants en fonction du fait qu’ils avaient déjà travaillé par quarts ou non. Afin de caractériser le type de travail effectué par ces travailleurs de quarts, nous avons décrit le milieu de travail pour chaque emploi de jour, de soir ou nuit, par quarts ou selon une autre formule de rotation.

Nous avons estimé les rapports de cote (RC) et les intervalles de confiance (IC) à $95 \%$ pour les associations entre le travail par quarts et le surpoids ainsi que l'obésité en effectuant une régression logistique polytomique multivariée et en utilisant le groupe ayant un poids normal comme référence ${ }^{32}$. Nous avons exploré les diverses associations pouvant exister entre le fait d'avoir déjà travaillé par quarts et l'obésité en caractérisant le travail par quarts selon trois modèles statistiques distincts: le fait d'avoir travaillé selon des quarts permanents de soir ou nuit, par quarts en rotation ou selon une autre formule de quarts. Nous avons seulement pu examiner les répercussions de la durée du travail par quarts sur les personnes travaillant par quarts en rotation, car la puissance statistique n'était pas suffisante dans le cas des personnes travaillant selon un quart permanent de soir ou nuit. Nous avons classé la durée du travail par quarts en trois catégories (jusqu'à 14 ans, de 15 à 29 ans, 30 ans et plus), afin de distinguer les personnes ayant travaillé par quarts pendant une brève période, pendant une période moyenne et pendant une longue période. Ces catégories ont été utilisées antérieurement pour établir des associations entre le travail par quarts et le cancer ${ }^{33-36}$.

Nous avons sélectionné les variables de confusion au moyen d'un graphe acyclique dirigé (GAD). Les GAD sont des diagrammes de causalité qui illustrent le sens des relations entre les variables d'intérêt et des variables de confusion inconnues. Ces graphes ont été proposés comme solution de rechange aux méthodes épidémiologiques classiques visant à définir les variables de confusion $^{37}$, car ils facilitent le processus de détermination des causes et en offre une représentation explicite ${ }^{38}$. En effet, contrairement aux modèles élaborés selon des méthodes statistiques, les GAD utilisés pour identifier les variables de confusion potentielles reposent sur les relations causales théoriques entre variables ${ }^{37}$. On s'en sert en épidémiologie pour déterminer, à partir de la relation causale posée comme hypothèse, un ensemble (minimalement suffisant) de variables de confusion qui permette le contrôle des confusions possibles entre un type d'exposition et un résultat ${ }^{37}$. Nous avons utilisé le logiciel DAGitty $^{39, *}$ pour créer un GAD et trouver un ensemble d'ajustements minimalement suffisant pour l'association entre le travail par quarts et le surpoids et l'obésité (figure 1). Cet ensemble comprend l'âge et le statut socio-économique (caractérisé par le niveau de scolarité et le revenu familial), et nous avons ajusté tous les modèles multivariés en fonction de ces variables. Nous avons mené toutes les analyses à l'aide de la version $9.2 \mathrm{du}$ logiciel SAS (SAS Institute Inc., Cary, Caroline du Nord, États-Unis).

\section{Résultats}

Chez les hommes ayant déjà travaillé par quarts, la proportion de travailleurs de scolarité de niveau postsecondaire ou de formation universitaire supérieure était plus faible que chez les hommes n'ayant jamais travaillé par quarts. De même, une plus grande proportion d'hommes ayant déjà travaillé par quarts avaient un revenu familial correspondant aux deux catégories inférieures, étaient fumeurs ou avaient déjà fumé, ou avaient déjà occupé un emploi exigeant des efforts physiques modérés à intenses pendant trois ou quatre périodes de vie (tableau 1).

En matière de statut pondéral, une plus grande proportion d'hommes de poids normal avait une scolarité de niveau postsecondaire ou une formation universitaire supérieure (25\%) par rapport aux hommes en surpoids (19\%) ou obèses (16\%). De plus, la proportion d'hommes en surpoids ou obèses ayant indiqué avoir occupé un emploi exigeant des efforts physiques modérés ou intenses au cours de trois ou quatre périodes de vie (en surpoids : $49 \%$, obèses : $48 \%$ ) était supérieure à la proportion d'hommes de poids normal (43\%) ayant occupé ce type d'emploi. Inversement, les proportions pour les deux quartiles supérieurs d'activité physique récréative étaient similaires chez les hommes de poids normal (54\%) et les hommes

*www.dagitty.net 
FIGURE 1

Graphe acyclique dirigé représentant l'association entre le travail par quarts et l'obésité, produit au moyen du logiciel DAGittya

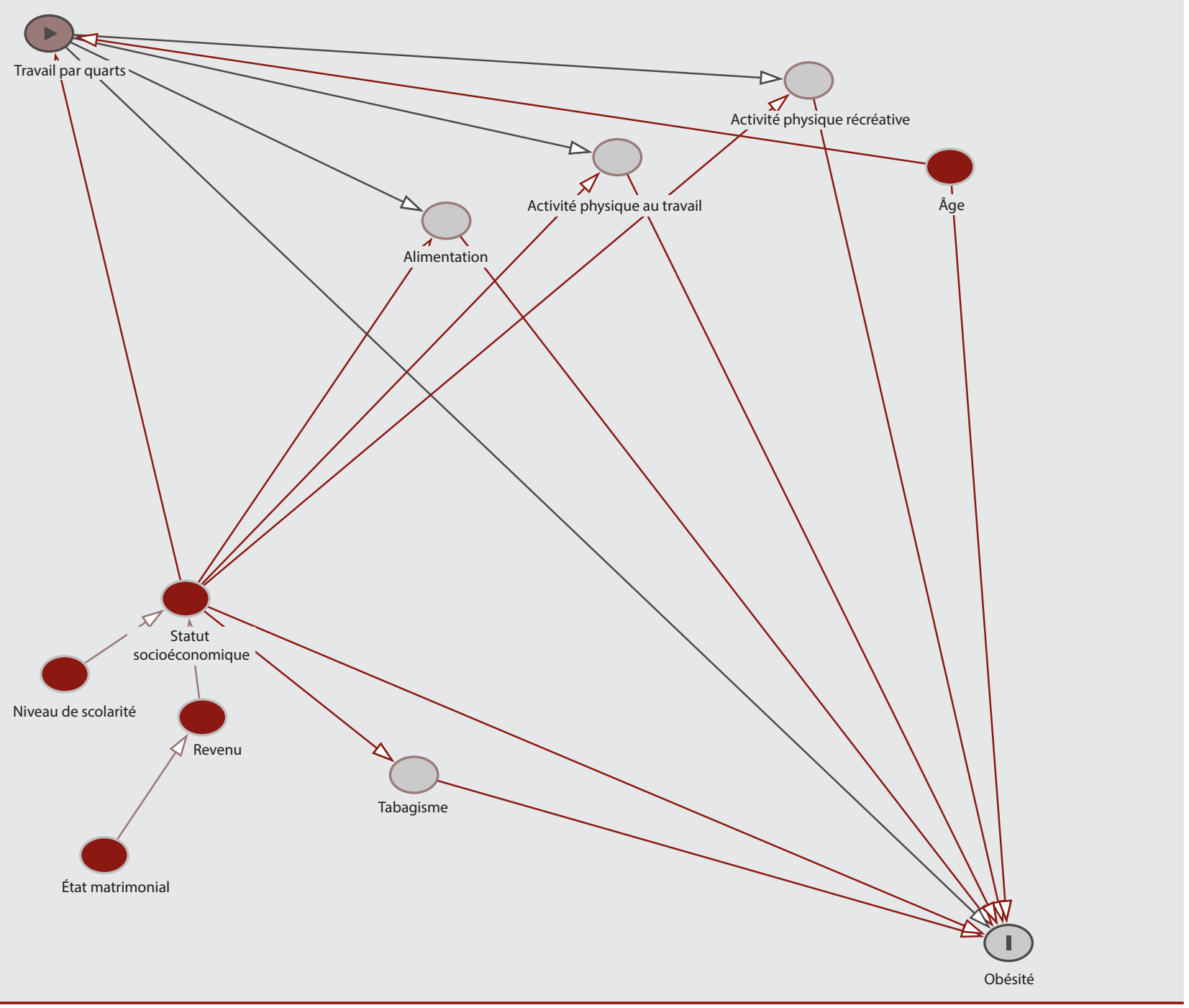

${ }^{a}$ DAGitty $^{39}$ : logiciel permettant de dessiner et d'analyser des schémas de causalité, voir www.dagitty.net.

accusant un surpoids (53\%), mais légèrement inférieures chez les hommes obèses (47 \%). Il n'y avait toutefois aucune différence majeure en ce qui concerne la consommation énergétique totale : $41 \%$ des hommes de poids normal ou en surpoids étaient classés dans les deux quartiles supérieurs de la consommation énergétique, pour $44 \%$ des hommes obèses (tableau 1).

Le tableau 2 présente la fréquence de divers types de quarts pour dix catégories de milieux de travail. Le type de quart le moins fréquent était le travail de soir ou nuit, et la plus grande proportion d'hommes travaillant selon ce quart (11 \%) œuvrait dans le domaine de la restauration ou de l'hôtellerie. Le travail par quarts en rotation s'est révélé la formule la plus fréquente, les proportions les plus élevées étant observées chez les hommes travaillant dans des mines (63\%), dans des usines (49\%) ou en laboratoire (26\%). Les autres formules de quarts étaient plus fréquentes chez les hommes travaillant en restauration et hôtellerie (37 \%) ou dans un véhicule $(22 \%)$.

Après exclusion de 61 hommes pour lesquels nous ne disposions pas d'information sur l'IMC, nous avons effectué une analyse multivariée sur notre échantillon de 1561 hommes. Nous avons observé une association entre le travail par quarts et le surpoids $(\mathrm{RC}=1,34$; IC à $95 \%: 1,05$ à 1,73 ) ainsi que l'obésité ( $\mathrm{RC}=1,57$, IC à $95 \%: 1,12$ à 2,21). De plus, bien que cela n'ait pas été statistiquement significatif, nous avons observé que, dans un contexte de travail de soir ou nuit, les rapports de cote associés aux hommes en surpoids $(\mathrm{RC}=1,12$, IC à $95 \%: 0,70$ à 1,79$)$ et aux hommes obèses ( $\mathrm{RC}=1,31$, IC à $95 \%: 0,71$ à 2,39) étaient élevés par rapport à ceux des hommes de poids normal (tableau 3). Nous n'avons observé aucune association entre les autres formules de travail par quarts et l'obésité. Bien que les rapports de cote aient été généralement plus élevés chez les personnes obèses que chez les personnes en surpoids, les tests de tendance pour l'ensemble des catégories ne se sont pas révélés statistiquement significatifs (données non présentées).

Nous avons classé la durée du travail par quarts en quatre catégories : aucune année, jusqu'à 14 ans, de 15 à 29 ans et 30 ans ou plus. Nous avons observé une association 
TABLEAU 1

Caractéristiques de la population à l'étude en fonction de son statut pondéral et de ses antécédents de travail par quarts, nord-est de l'Ontario, 1995 à $1998(n=1561)^{\mathrm{a}}$

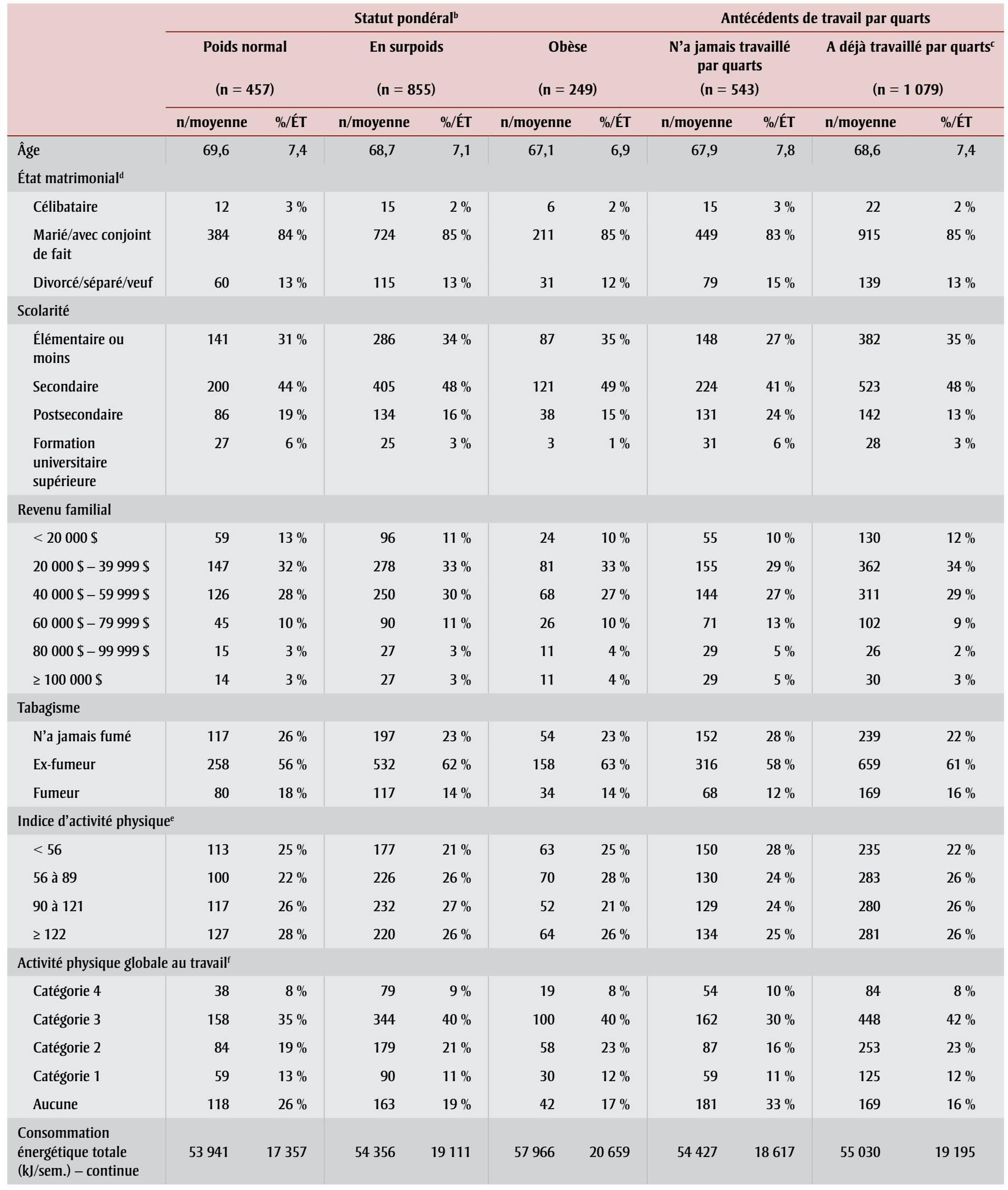




\section{TABLEAU 1 (suite) \\ Caractéristiques de la population à l'étude en fonction de son statut pondéral et de ses antécédents de travail par quarts, nord-est de l'Ontario, 1995 à $1998(n=1561)^{a}$}

\begin{tabular}{|c|c|c|c|c|c|c|c|c|c|c|}
\hline & \multicolumn{6}{|c|}{ Statut pondéral ${ }^{b}$} & \multicolumn{4}{|c|}{ Antécédents de travail par quarts } \\
\hline & \multicolumn{2}{|c|}{$\begin{array}{l}\text { Poids normal } \\
\qquad(n=457)\end{array}$} & \multicolumn{2}{|c|}{$\begin{array}{l}\text { En surpoids } \\
\qquad(\mathbf{n}=\mathbf{8 5 5})\end{array}$} & \multicolumn{2}{|c|}{$\begin{array}{l}\text { Obèse } \\
\text { (n= 249) }\end{array}$} & \multicolumn{2}{|c|}{$\begin{array}{l}\text { N'a jamais travaillé } \\
\text { par quarts } \\
(n=543)\end{array}$} & \multicolumn{2}{|c|}{$\begin{array}{l}\text { A déjà travaillé par quarts } \\
\qquad(n=1079)\end{array}$} \\
\hline & n/moyenne & \%/ÉT & n/moyenne & \%/ÉT & n/moyenne & \%/ÉT & n/moyenne & \%/ÉT & n/moyenne & \%/ÉT \\
\hline \multicolumn{11}{|c|}{ Consommation énergétique totale $(\mathrm{kJ} / \mathrm{sem}$.) - par catégories } \\
\hline 54785 à 66330 & 93 & $20 \%$ & 193 & $23 \%$ & 50 & $20 \%$ & 102 & $19 \%$ & 138 & $22 \%$ \\
\hline 66331 ou plus & 96 & $21 \%$ & 152 & $18 \%$ & 59 & $24 \%$ & 106 & $20 \%$ & 209 & $19 \%$ \\
\hline Inconnue & 29 & $6 \%$ & 58 & $7 \%$ & 16 & $6 \%$ & 62 & $11 \%$ & 64 & $6 \%$ \\
\hline
\end{tabular}

Abréviations : ÉT, écart type; kJ/sem., kilojoules par semaine.

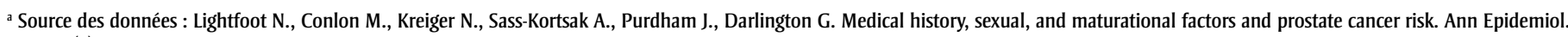
2004;14(9):655-662.

${ }^{\mathrm{b}}$ Aucune information disponible sur l'IMC pour 61 personnes.

c Sujets ayant déjà travaillé par quarts (de soir ou nuit, par quarts en rotation ou selon une autre formule de quarts).

d L'état matrimonial de trois participants ( 1 au poids normal, 1 en surpoids et 1 obèse, aucun d'entre eux n'ayant travaillé par quarts) n'a pas été fourni.

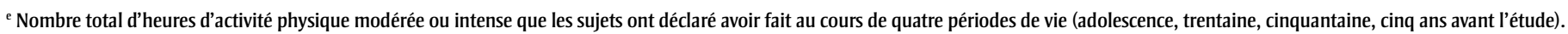

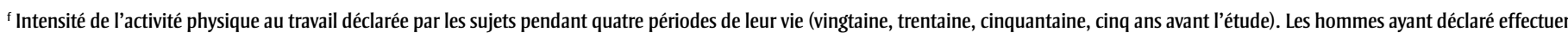

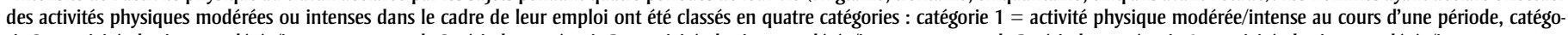

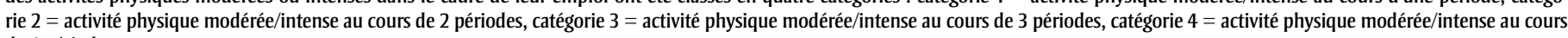
de 4 périodes.

statistiquement significative entre le fait d'avoir travaillé par quarts pendant 30 ans ou plus et l'obésité ( $\mathrm{RC}=1,86$, IC à $95 \%$ : $1,16$ à 2,96$)$. Les rapports de cote associés à l'obésité étaient élevés chez les hommes ayant travaillé par quarts jusqu'à 14 ans et entre 15 et 29 ans et pour les hommes en surpoids dans les trois catégories de durée (tableau 4). Tant dans le groupe des personnes en surpoids $(p=0,03)$ que dans celui des personnes obèses ( $p=0,008)$, nous avons observé une tendance statistiquement significative en fonction de la durée de travail par quarts.
Nous avons effectué une analyse de sensibilité en excluant tous les hommes dont l'IMC avait été calculé en fonction de leur taille au début de la trentaine ou de leur poids au début de la cinquantaine ( $n=189$ sujets). Nos résultats se sont révélés très semblables à ceux observés sur l'ensemble complet de données (données non présentées), tant pour le fait d'avoir déjà travaillé par quarts que pour la durée du travail par quarts, et tant pour les quarts de soir ou nuit que pour les autres formules de travail par quarts.

\section{Analyse}

Dans cette étude menée auprès de plus de 1500 hommes, nous avons montré que le travail par quarts est associé à un risque accru de surpoids ou d'obésité. Le travail selon un quart permanent de soir ou nuit s'est révélé également associé à un risque accru de surpoids ou d'obésité, mais ces résultats n'étaient pas statistiquement significatifs. Les études antérieures ayant porté sur le travail par quarts et l'obésité ont été essentiellement menées au sein

TABLEAU 2

Milieux de travail pour chaque emploi, en fonction du type de quart de travail, nord-est de l'Ontario, 1995 à 1998 (n = 1561$)^{\text {a }}$

\begin{tabular}{|c|c|c|c|c|c|c|c|c|c|c|c|c|c|c|c|c|c|c|c|c|}
\hline \multirow{3}{*}{$\begin{array}{l}\text { Type de } \\
\text { quart de } \\
\text { travail }\end{array}$} & \multicolumn{20}{|c|}{ Milieu de travail } \\
\hline & \multicolumn{2}{|c|}{$\begin{array}{c}\text { Mine } \\
(n=790)\end{array}$} & \multicolumn{2}{|c|}{$\begin{array}{c}\text { Usine } \\
(n=1426)\end{array}$} & \multicolumn{2}{|c|}{$\begin{array}{l}\text { Laboratoire } \\
\qquad(\mathbf{n}=73)\end{array}$} & \multicolumn{2}{|c|}{$\begin{array}{l}\text { Véhicule } \\
(n=730)\end{array}$} & \multicolumn{2}{|c|}{$\begin{array}{c}\text { Restaurant/ } \\
\text { hôtel } \\
(n=107)\end{array}$} & \multicolumn{2}{|c|}{$\begin{array}{l}\text { Entrepôt } \\
(n=179)\end{array}$} & \multicolumn{2}{|c|}{$\begin{array}{l}\text { Extérieur } \\
(\mathrm{n}=1425)\end{array}$} & \multicolumn{2}{|c|}{$\begin{array}{c}\text { Chantier de } \\
\text { construction } \\
(n=433)\end{array}$} & \multicolumn{2}{|c|}{$\begin{array}{c}\text { Bureau } \\
(n=1370)\end{array}$} & \multicolumn{2}{|c|}{$\begin{array}{c}\text { Autre } \\
(n=1623)\end{array}$} \\
\hline & $\mathbf{n}$ & $\%$ & $\mathbf{n}$ & $\%$ & $\mathbf{n}$ & $\%$ & $\mathbf{n}$ & $\%$ & $\mathbf{n}$ & $\%$ & n & $\%$ & $\mathbf{n}$ & $\%$ & n & $\%$ & $\mathbf{n}$ & $\%$ & $\mathbf{n}$ & $\%$ \\
\hline Jour & 137 & 17 & 624 & 44 & 53 & 73 & 394 & 54 & 35 & 33 & 132 & 74 & 1019 & 86 & 373 & 86 & 1177 & 86 & 1108 & 68 \\
\hline En rotation & 495 & 63 & 693 & 49 & 19 & 26 & 146 & 20 & 19 & 18 & 31 & 17 & 148 & 10 & 39 & 9 & 70 & 5 & 235 & 14 \\
\hline Autre & 121 & 15 & 71 & 5 & 0 & 0 & 163 & 22 & 40 & 37 & 9 & 5 & 234 & 16 & 16 & 4 & 94 & 7 & 222 & 14 \\
\hline
\end{tabular}

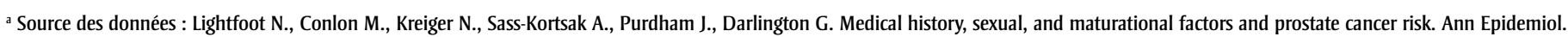
2004;14(9):655-662. 
TABLEAU 3

Associations entre le travail par quarts ${ }^{\mathrm{a}}$ et le surpoids et l'obésité, nord-est de l'Ontario, 1995 à 1998 (n = 1561$)^{\text {b }}$

\begin{tabular}{|c|c|c|c|c|c|c|c|c|c|c|c|}
\hline \multirow[b]{2}{*}{$\begin{array}{l}\text { Types de travail } \\
\text { par quarts }\end{array}$} & \multicolumn{3}{|c|}{$\begin{array}{l}\text { Poids normal } \\
\quad(n=457)\end{array}$} & \multicolumn{4}{|c|}{$\begin{array}{l}\text { Surpoids } \\
(\mathrm{n}=\mathbf{8 5 5})\end{array}$} & \multicolumn{4}{|c|}{$\begin{array}{l}\text { Obésité } \\
(n=249)\end{array}$} \\
\hline & $\mathbf{n}$ & $\%$ & $\begin{array}{c}\text { RC }^{\text {d }} \\
(\text { IC à } 95 \%)\end{array}$ & $\mathbf{n}$ & $\%$ & $\begin{array}{c}\mathbf{R C}^{\mathbf{b}} \\
(\mathrm{IC} \text { à } 95 \%)\end{array}$ & $\begin{array}{c}\mathrm{RC}^{\mathrm{e}} \\
(\mathrm{IC} \text { à } 95 \%)\end{array}$ & $\mathbf{n}$ & $\%$ & $\begin{array}{c}\mathbf{R C}^{\mathbf{b}} \\
(\mathrm{IC} \text { à } 95 \%)\end{array}$ & $\begin{array}{c}\mathrm{RC}^{\mathrm{e}} \\
(\mathrm{IC} \text { à } 95 \%)\end{array}$ \\
\hline Travail de soir/nuit & 30 & 7 & 1,00 (réf.) & 67 & 8 & $1,21(0,77$ à 1,89$)$ & $1,12(0,70$ à 1,79$)$ & 22 & 9 & $1,38(0,78$ à 2,45$)$ & $1,31(0,71$ à 2,39$)$ \\
\hline Travail par quarts & 178 & 39 & 1,00 (réf.) & 410 & 48 & $1,44(1,15$ à 1,82$)$ & $1,34(1,05$ à 1,73$)$ & 135 & 53 & $1,77(1,30$ à 2,42$)$ & $1,57(1,12$ à 2,21$)$ \\
\hline Autre & 136 & 30 & 1,00 (réf.) & 252 & 29 & $0,99(0,77$ à 1,27$)$ & $0,98(0,75$ à 1,28$)$ & 71 & 29 & $0,94(0,67$ à 1,32$)$ & $0,97(0,67$ à 1,41$)$ \\
\hline
\end{tabular}

Abréviations : IC, intervalle de confiance; RC, rapport de cote; réf., référence.

${ }^{a}$ Hommes ayant déclaré avoir déjà travaillé de soir ou de nuit, par quarts en rotation ou selon une autre formule de quarts.

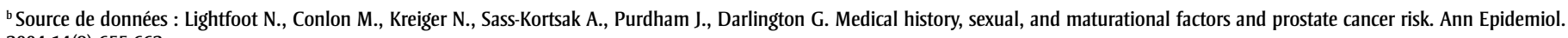
2004;14(9):655-662.

' Les catégories de travail par quarts (de nuit ou par quarts en rotation, etc.) ne sont pas mutuellement exclusives.

${ }^{\mathrm{d}}$ Modèle non ajusté.

${ }^{\text {e }}$ Modèle ajusté en fonction de l'âge, de la scolarité et du revenu familial.

d'une même entreprise ou industrie, seul un faible nombre d'entre elles comparant directement, comme nous l'avons fait dans cette étude, les effets du travail selon un quart permanent ou des quarts par rotation au sein d'une même population. La plupart des études ayant porté sur le travail par quarts et l'obésité étaient axées sur le travail par quarts et ont révélé, de façon analogue à notre étude, l'existence d'une association positive statistiquement significative entre le travail par quarts et l'obésitéf,17,19,20,22,27. Il convient toutefois de préciser que certaines études ne corroborent pas l'existence d'une telle association $^{10,21}$. Par ailleurs, peu d'études ont porté expressément sur les effets du travail selon un quart de nuit permanent. Une étude transversale a mis en lumière l'existence d'un lien entre le travail selon un quart de nuit permanent et une prise pondérale chez le personnel infirmier et le personnel de sécurité , et le travail selon un quart de nuit permanent a été associé à une prévalence accrue de l'obésité chez des personnes travaillant dans des établissements avicoles $^{14}$ et chez des femmes travaillant dans une usine de fabrication de semi-conducteurs ${ }^{15}$.

Nous avons observé une tendance à la hausse du risque de surpoids et d'obésité en fonction du temps passé à travailler par quarts. Bien que les rapports de cote aient été élevés pour toutes les catégories de quarts, l'association la plus forte et la plus statistiquement significative portait sur le travail par quarts pendant 30 ans ou plus et l'obésité. Peu d'études antérieures ont tenu compte du temps passé à travailler par quarts au moment d'évaluer l'association avec l'obésité. Chez les femmes ayant participé à l'étude Million Women Study, on a observé une tendance à la hausse du risque d'obésité en fonction du temps passé à travailler par quarts ${ }^{18}$. La durée du travail par quarts a également été considérée comme un prédicteur de l'IMC chez des travailleurs du secteur pétrolier et gazier $^{8}$, et comme un prédicteur du rapport taille-hanches chez des femmes travaillant comme infirmières en milieu hospitalier et chez des hommes travaillant en usine ${ }^{16}$. Dans une étude utilisant une définition nominale de la durée semblable à la nôtre, les chercheurs ont observé une relation positive entre le rapport taille-hanches et le fait d'avoir travaillé par quarts pendant 2 à 5 ans et pendant 5 ans ou plus, et entre l'IMC et le fait d'avoir travaillé par quarts pendant 5 ans chez des travailleurs de sexe masculin $^{22}$. Il convient toutefois de préciser que toutes les études n’ont pas révélé une

TABLEAU 4

Association entre la durée du travail par quarts et le surpoids ainsi que l'obésité, nord-est de l’Ontario, 1995 à 1998 (n = 1561$)^{a}$

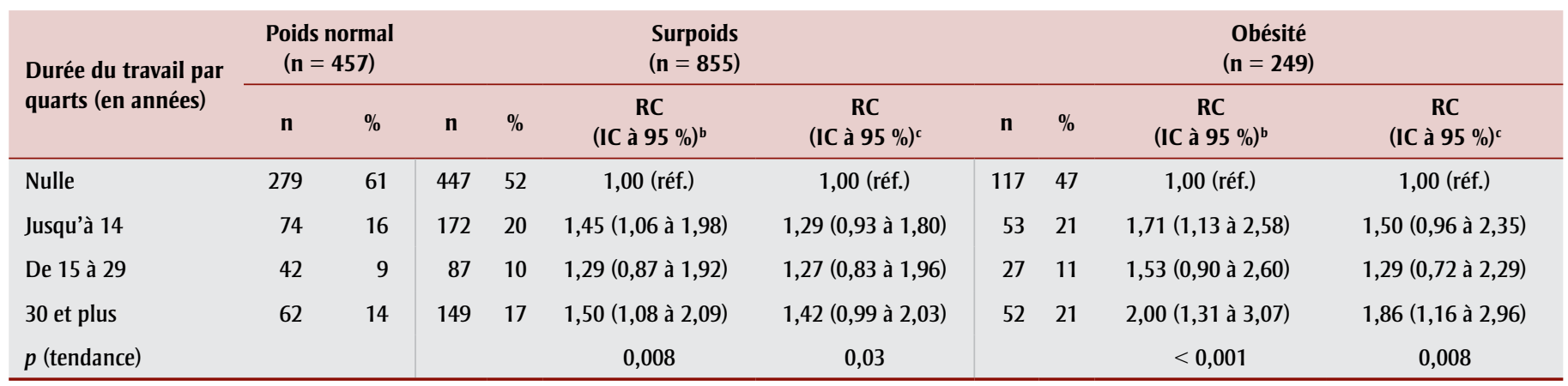

Abréviations : IC, intervalle de confiance; RC, rapport de cote; réf., référence.

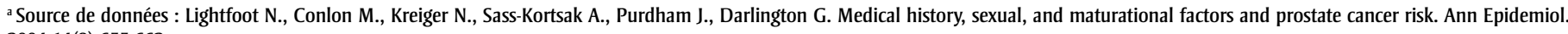
2004;14(9):655-662.

${ }^{\mathrm{b}}$ Modèle non ajusté.

' Modèle ajusté en fonction de l'âge, de la scolarité et du revenu familial. 
association positive avec l'obésité. Par exemple, selon une étude transversale menée auprès d'hommes de 35 à 60 ans, le temps passé à travailler par quarts n'aurait aucune incidence ${ }^{7}$. Comme dans notre analyse, des études ont examiné l'incidence de la durée du travail par quarts sur l'obésité de façon transversale $7,8,16,18,22$, mais aucune relation temporelle définitive n'a pu être établie entre le travail par quarts et l'obésité.

La relation entre la durée du travail par quarts et l'obésité est intéressante car l'obésité constituerait un jalon intermédiaire entre le travail par quarts et d'autres problèmes de santé, en particulier le cancer. Plus particulièrement, plusieurs études portant sur la relation entre le travail par quarts et le cancer du sein chez les femmes ont révélé une augmentation du risque de cancer du sein chez celles travaillant par quarts pendant une longue période ${ }^{1,40,41}$. Selon une analyse antérieure de l'étude cas-témoins sur le cancer de la prostate, dont les données ont servi de point de départ pour notre analyse, il existe une association entre le fait d'avoir travaillé par quarts pendant sept ans ou moins et le risque de cancer de la prostate, mais aucune association pour les périodes supérieures à sept $a n s^{42}$ et, selon une étude canadienne menée en 2012 , le risque de cancer de la prostate est accru pour les périodes de moins de cinq ans, de cinq à dix ans et de dix ans ou plus passées à travailler par quarts $^{43}$. Par ailleurs, selon des résultats obtenus récemment en Espagne, il existerait une augmentation statistiquement non significative du risque de cancer de la prostate chez les hommes travaillant de nuit de façon permanente ou par quarts depuis 28 ans ou plus ${ }^{44}$. Aucune étude portant expressément sur les relations entre le travail par quarts, l'obésité et le cancer dans un contexte longitudinal n'a été menée.

On a proposé plusieurs mécanismes d'explication par lesquels l'exposition au travail par quarts pourrait être associée à l'obésité, la plupart concernant les perturbations du rythme circadien. Par exemple, l'efficacité métabolique variant selon le moment de la journée auquel on consomme de la nourriture, le fait que les personnes qui travaillent de nuit prennent leurs repas à des heures différentes pourrait constituer un mécanisme par lequel le travail par quarts influerait sur l'obésité ${ }^{45}$. Les perturbations du sommeil peuvent avoir également jouer sur le métabolisme, et plusieurs études ont montré que les personnes qui travaillent de nuit dorment généralement moins longtemps ${ }^{2,18,46,47}$. Bien que nous ayions présenté des données sur la consommation énergétique, nous ne disposions dans cette étude d'aucune information sur l'heure des repas ou sur les modalités du sommeil (par exemple sa durée), de sorte qu'il s'est avéré impossible d'évaluer les effets de ces mécanismes.

\section{Forces et limites}

L'une des forces de cette étude est le fait qu'elle ait été menée à l'échelle d'une population, ce qui permet de rendre compte de multiples types de travail par quarts au sein d'une même population. En comparaison des études antérieures menées au sein d'une même industrie ou dans un même lieu de travail, et ne portant que sur un type de travail par quarts $7,8,10-15,17,19,20,23,24,26,27$, notre analyse a révélé que les relations avec l'obésité étaient plus étroites dans le cas des personnes travaillant par quarts en rotation que dans celui des personnes travaillant de soir ou nuit de façon permanente. Notre analyse tient également compte de l'incidence de plusieurs facteurs de confusion potentiels sur la relation entre le travail par quarts et l'obésité, ce qui constitue une amélioration par rapport aux études antérieures, auxquelles on a notamment reproché, dans un article de synthèse publié en 2011, une prise en considération insuffisante des facteurs de confusion potentiels $^{28}$. Enfin, nous avons utilisé dans notre étude les antécédents professionnels des participants sur l'ensemble de leur vie pour caractériser le travail par quarts, ce qui permet d'évaluer l'exposition de façon plus exhaustive que dans bon nombre d'études antérieures, où il n'a été tenu compte que de l'emploi alors occupé par les participants ou de leurs antécédents professionnels au sein d'une même entreprise $\mathrm{e}^{7-11,13-17,19-25,27}$.

Malgré ces forces, notre étude présente certaines limites. Bien que l'utilisation d'un ensemble de données fondé sur une population nous ait permis de tenir compte, dans une même étude, d'un plus grand nombre de types de travail par quarts (quart de soir ou nuit permanent, par quarts en rotation) que dans la plupart des travaux de recherche antérieurs ${ }^{7-27}$, nous ne possédions aucune information détaillée sur les caractéristiques de ces quarts (p. ex. structure des rotations, nombre de nuits consécutives, rotation avant ou rotation arrière). De plus, dans le cadre de notre analyse, nous avons jumelé les quarts de soir et les quarts de nuit ainsi que toutes les autres formules de travail par quarts pouvant englober un quart de soir ou un quart de nuit. En 2009, un groupe de travail du Centre international de Recherche sur le Cancer $(\mathrm{CIRC})^{48}$ a indiqué à quel point il était important d'avoir de l'information détaillée sur les caractéristiques des quarts de travail pour effectuer une évaluation de l'exposition, de sorte qu'il faudra mener davantage de travaux tenant compte de ces caractéristiques pour examiner les liens avec l'obésité. Si des quarts différents produisent des perturbations différentes du rythme circadien, le fait de jumeler des types de quarts, comme nous l'avons fait dans notre analyse, pourrait de fait entraîner une confusion résiduelle.

De longues heures de travail ont également été associées à un risque d'obésitét ${ }^{49}$, mais nous ne possédions aucunes données sur le sujet. De plus, bien que notre étude ait porté sur environ 1500 hommes, la proportion d'hommes exposés à un quart de soir ou nuit dans chaque catégorie de poids était relativement faible $(<10 \%)$. En raison de la petite taille de l'échantillon, et puisque nous ne pouvions tenir compte de la durée de ce type de travail par quarts, il est possible que notre étude soit limitée sur le plan de la puissance de détection d'associations réelles entre le travail permanent de soir ou nuit et l'obésité. Des études ultérieures portant sur un plus grand nombre de personnes travaillant selon un quart permanent de soir ou nuit nous permettront de mieux examiner ces relations. Par ailleurs, les milieux de travail pourraient induire une confusion résiduelle dans la mesure où certaines de leurs caractéristiques autres que le travail par quarts constitueraient les véritables facteurs de risque d'obésité. Cependant, étant donné que les résultats de notre étude montrant que le travail par quarts accroît le risque d'obésité concordent avec ceux des travaux de recherche menés dans divers milieux de travail $1^{7,17,19,20,22,27}$, il semble plus vraisemblable que la relation observée entre le travail par quarts et l’obésité soit réelle.

L'utilisation de données autodéclarées sur la taille et le poids pour déterminer l'IMC comporte un risque d'erreur de classification, car la surestimation de la taille et la sous-estimation du poids peuvent entraîner une sous-estimation de l'IMC ${ }^{50}$. Des erreurs de classification de l'IMC pourraient avoir réduit la précision des estimations 
concernant l'association entre le travail par quarts, le surpoids et l'obésité, car des participants qui accusent en fait un surpoids ou sont obèses pourraient avoir été inclus dans le groupe de participants au poids normal. Il est à noter, toutefois, que la proportion d'hommes considérés comme étant en surpoids ou obèses dans notre étude $(70,7 \%)$ est semblable à la proportion d'hommes en surpoids ou obèses de 45 ans ou plus $(68,0 \%)$ calculée par Statistique Canada en 2000 pour le nord-est de l'Ontario ${ }^{51}$.

Des données autodéclarées ont également été utilisées pour l'alimentation, l'activité physique générale et l'activité physique au travail, de sorte qu'il pourrait aussi y avoir, dans une certaine mesure, une erreur de classification. Cependant, comme ces variables ont été utilisées à des fins descriptives dans la population à l'étude et qu'elles n’ont pas été incluses dans les modèles multivariés utilisés pour évaluer la relation entre le travail par quarts et l'obésité, il est peu probable qu'une erreur de classification ait influé sur les relations observées.

Le taux de réponse relativement faible des témoins dans cet ensemble de données $(47,5$ \%) a pu entraîner un biais de sélection, surtout si la participation à l'étude s'est trouvée liée aux antécédents de travail par quarts et au statut pondéral. Toutefois, la proportion d'hommes ayant des antécédents de travail par quarts dans notre étude (33 \%) est semblable à la proportion de Canadiens travaillant par quarts selon l'Enquête sur la dynamique du travail et du revenu menée par Statistique Canada en $2005^{52}$, ce qui laisserait entendre que le travail par quarts lui-même n'a pas été un déterminant majeur de la participation à l'étude. Bien que l'on puisse soupçonner que la proportion réelle de travailleurs par quarts dans le nord-est de l'Ontario soit plus élevée que la moyenne canadienne, impliquant une sous-représentation des travailleurs par quarts dans notre étude, nous pensons peu probable, étant donné que la participation à l'étude ne semble pas avoir été liée au statut pondéral, que l'association entre le travail par quarts et la participation à l'étude constitue un biais de sélection réel.

Enfin, comme cette étude a été menée chez des hommes presque exclusivement de race blanche ${ }^{30}$, ces résultats pourraient ne pas être généralisables à d'autres sous-groupes, par exemple les femmes ou les hommes d'autre origine ethnique.

\section{Conclusion}

Notre étude a révélé qu'il existait une association positive entre le travail par quarts et le surpoids ainsi que l'obésité et laisse entendre qu'il existerait également une association en ce qui concerne le travail de soir ou nuit. Nous avons également observé une association entre une durée croissante de travail par quarts et l'obésité, surtout dans le cas du travail par quarts sur une longue période. Si un certain nombre d'études antérieures ont soutenu l'existence d'une relation entre le travail par quarts et l'obésité , $7,13-15,17-20,26,27$, d'autres travaux de recherche en population, comme notre étude, permettent de mieux saisir dans quelle mesure certaines formules de travail par quarts ont une plus grande incidence sur l'obésité que d'autres. Étant donné que le travail par quarts est constitutif de bon nombre d'emplois, savoir quelles formules de travail par quarts ont une incidence négative sur la santé, par exemple en association avec l'obésité, est nécessaire pour élaborer des politiques sur l'organisation optimale des quarts de travail dans le but de préserver la santé des travailleurs.

\section{Remerciements}

Létude cas-témoins sur le cancer de la prostate a été financée par des subventions de recherche du Programme national de recherche et de développement en matière de santé (numéro de projet 66065574-502), de la Fondation du Nord en cancérologie et du Canadian Union of the Mine, Mill and Smelter Workers - section locale 598. Anne Grundy a reçu une bourse de recherche postdoctorale d'Action Cancer Ontario pour mener cette étude.

\section{Conflits d'intérêts}

Les auteurs déclarent n'avoir aucun conflit d'intérêts.

\section{Contribution des auteurs}

NL était chercheure principale responsable de la Men's Health Study, l'étude castémoins sur le cancer de la prostate dont les données ont été utilisées pour cette analyse. La conception de l'analyse du travail par quarts, l'analyse statistique, l'interprétation des résultats et la rédaction du manuscrit ont été effectuées par AG, avec une rétroaction de la part de $\mathrm{MC}, \mathrm{VK}$, VN, NL et NK.

\section{Références}

1. Wang X-S, Armstrong MEG, Cairns BJ, Key TJ, Travis RC. Shift work and chronic disease: the epidemiological evidence. Occup Med (London). 2011; 61(2):78-89.

2. Pan A, Schernhammer ES, Sun Q, Hu FB. Rotating night shift work and risk of type 2 diabetes: two prospective cohort studies in women. PLoS Med. 2011;8(12):e1001141. doi: 10.1371 /journal.pmed.1001141.

3. Morikawa Y, Nakagawa H, Miura K, et al. Shift work and the risk of diabetes mellitus among Japanese male factory workers. Scand J Work Environ Health. 2005;31(3):179-183.

4. Karlsson B, Knutsson A, Lindahl B. Is there an association between shift work and having a metabolic syndrome? Results from a population based study of 27485 people. Occup Environ Med. 2001;58(11):747-752.

5. De Bacquer D, Van Risseghem $M$, Clays E, Kittel F, De Backer G, Braeckman L. Rotating shift work and the metabolic syndrome: a prospective study. Int J Epidemiol. 2009; 38(3):848-854.

6. Straif K, Baan R, Grosse Y, et al. Carcinogenicity of shift-work, painting, and fire-fighting. Lancet Oncol. 2007;8(12):1065-1066.

7. Di Lorenzo L, De Pergola G, Zocchetti C, et al. Effect of shift work on body mass index: results of a study performed in 319 glucose-tolerant men working in a Southern Italian industry. Int J Obes Relat Metab Disord. 2003;27(11):1353-1358.

8. Parkes KR. Shift work and age as interactive predictors of body mass index among offshore workers. Scand J Work Environ Health. 2002;28(1): 64-71.

9. Geliebter A, Gluck ME, Tanowitz M, Aronoff NJ, Zammit GK. Work-shift period and weight change. Nutrition. 2000 Jan;16(1):27-29. 
10. Nakamura K, Shimai S, Kikuchi S, et al. Shift work and risk factors for coronary heart disease in Japanese bluecollar workers: serum lipids and anthropometric characteristics. Occup Med (Lond). 1997;47(3):142-146.

11. Ghiasvand M, Heshmat R, Golpira R, et al. Shift working and risk of lipid disorders: a cross-sectional study. Lipids Health Dis. 2006;5:9. doi : 10.1186/1476-511X-5-9.

12. Karlsson BH, Knutsson AK, Lindahl BO, Alfredsson LS. Metabolic disturbances in male workers with rotating three-shift work. Results of the WOLF study. Int Arch Occup Environ Health. 2003;76(6):424-430.

13. Ishizaki M, Morikawa $Y$, Nakagawa $H$, et al. The influence of work characteristics on body mass index and waist to hip ratio in Japanese employees. Ind Health. 2004;42(1):41-49.

14. Macagnan J, Pattussi MP, Canuto R, Henn RL, Fassa AG, Olinto MT. Impact of nightshift work on overweight and abdominal obesity among workers of a poultry processing plant in southern Brazil. Chronobiol Int. 2012;29(3): 336-343.

15. Chen JD, Lin YC, Hsiao ST. Obesity and high blood pressure of 12-hour night shift female clean-room workers. Chronobiol Int. 2010;27(2):334-244.

16. Ha M, Park JP. Shiftwork and metabolic risk factors of cardiovascular disease. J Occup Health. 2005;47:89-95.

17. Manenschijn L, van Kruysbergen RGPM, de Jong FH, Koper JW, van Rossum EFC. Shift work at young age is associated with elevated long-term cortisol levels and body mass index. J Clin Endocrinol Metab. 2011;96(11): E1862-E1865.

18. Wang X-S, Travis RC, Reeves G, et al. Characteristics of the Million Women Study participants who have and have not worked at night. Scand J Work Environ Health. 2012;38(6):590-599.

19. Suwazono Y, Dochi M, Sakata K, et al. A longitudinal study on the effect of shift work on weight gain in male Japanese workers. Obesity (Silver Spring). 2008;16(8):1887-1893.
20. Morikawa Y, Nakagawa H, Miura K, et al. Effect of shift work on body mass index and metabolic parameters. Scand J Work Environ Health. 2007;33(1): 45-50.

21. van Amelsvoort LG, Schouten EG, Kok FJ. Impact of one year of shift work on cardiovascular disease risk factors. J Occup Environ Med. 2004;46(7): 699-706.

22. van Amelsvoort L, Schouten EG, Kok FJ. Duration of shiftwork related to body mass index and waist to hip ratio. Int J Obes Relat Metab Disord. 1999;23(9):973-978.

23. Sakata K, Suwazono Y, Harada H, Okubo Y, Kobayashi E, Nogawa K. The relationship between shift work and the onset of hypertension in male Japanese workers. J Occup Environ Med. 2003;45(9):1002-1006.

24. Yamada Y, Kameda M, Noborisaka Y. Excessive fatigue and weight gain in cleanroom workers after changing from an 8-hour to a 12-hour shift. Scand J Work Environ Health. 2001; 27(5):318-326.

25. Watari M, Uetani M, Suwazono Y, Kobayashi E, Kinouchi N, Nogawa K. A longitudinal study of the influence of smoking on the onset of obesity at a telecommunications company in Japan. Prev Med (Baltim). 2006;43(2):107-112.

26. Biggi N, Consonni D, Galluzzo V, Sogliani M, Costa G. Metabolic syndrome in permanent night workers. Chronobiol Int. 2008;25(2):443-454.

27. Kubo T, Oyama I, Nakamura T, et al. Retrospective cohort study of the risk of obesity among shift workers: findings from the Industry-based Shift Workers' Health study, Japan. Occup Environ Med. 2011;68(5):327-331.

28. van Drongelen A, Boot CRL, Merkus SL, Smid T, van der Beek AJ. The effects of shift work on body weight change - a systematic review of longitudinal studies. Scand J Work Environ Health. 2011;37(4):263-275.

29. Lightfoot $N$, Conlon $M$, Kreiger $N$, Sass-Kortsak A, Purdham J, Darlington G. Medical history, sexual, and maturational factors and prostate cancer risk. Ann Epidemiol. 2004;14(9): 655-662.
30. Darlington GA, Kreiger N, Lightfoot N, Purdham J, Sass-Kortsak A. Le risque de cancer de la prostate et l'alimentation, l'activité physique de loisir et le tabagisme. Maladies chroniques au Canada. 2007;27(4):158-167.

31. Organisation mondiale de la santé (OMS). Obésité : prévention et prise en charge de l'épidémie mondiale. Rapport d'une consultation de l'OMS, Genève, 3-5 juin 1997. Genève : OMS; 1998. 252 p.

32. Hosmer D, Lemeshow S. Special topics. Dans : Applied logistic regression. 2e éd. Toronto (Ont.) : John Wiley and Sons; 2000. p. 260-352.

33. Schernhammer ES, Laden F, Speizer $\mathrm{FE}$, et al. Rotating night shifts and risk of breast cancer in women participating in the nurses' health study. J Natl Cancer Inst. 2001;93(20):1563-1568.

34. Lie J-AS, Kjuus H, Zienolddiny S, Haugen A, Stevens RG, Kjærheim K. Night work and breast cancer risk among Norwegian nurses: assessment by different exposure metrics. Am J Epidemiol. 2011;173(11):1272-1279.

35. Hansen J, Lassen CF. Nested casecontrol study of night shift work and breast cancer risk among women in the Danish military. Occup Environ Med. 2012;69(8):551-556. doi: 10.1136 /oemed-2011-100240.

36. Grundy A, Richardson H, Burstyn I, et al. Increased risk of breast cancer associated with long-term shift work in Canada. Occup Environ Med. 2013; 70(12):831-838.

37. Greenland S, Pearl J, Robins JM. Causal diagrams for epidemiologic research. Epidemiology. 1999;10(1): $37-48$.

38. Szklo M, Nieto FJ. Epidemiology beyond the basics. 3e éd. Burlington (MA): Jones and Bartlett Learning; 2014. p. 162-163.

39. Textor J, Hardt J, Knüppel S. DAGitty: a graphical tool for analyzing causal diagrams. Epidemiology. 2011;22(5):745. 
40. Erren TC, Pape HG, Reiter RJ, Piekarski C. Chronodisruption and cancer. Naturwissenschaften. 2008;95(5):367382.

41. Wang F, Yeung KL, Chan WC, et al. A meta-analysis on dose-response relationship between night shift work and the risk of breast cancer. Ann Oncol. 2013;24(11):2724-2732.

42. Conlon $M$, Lightfoot $N$, Kreiger $N$. Rotating shift work and risk of prostate cancer. Epidemiology. 2007;18(1): 182-183.

43. Parent M-É, El-Zein M, Rousseau M-C, Pintos J, Siemiatycki J. Night work and the risk of cancer among men. Am J Epidemiol. 2012;176(9):751-759.

44. Papantoniou K, Castaño-Vinyals G, Espinosa A, et al. Night shift work, chronotype and prostate cancer risk in the MCC-Spain case-control study. Int J Cancer. 2015;137(5):1147-1157.

45. Antunes LC, Levandovski R, Dantas G, Caumo W, Hidalgo MP. Obesity and shift work: chronobiological aspects. Nutr Res Rev. 2010;23(1):155-168.

46. Grundy A, Sanchez M, Richardson H, et al. Light intensity exposure, sleep duration, physical activity, and biomarkers of melatonin among rotating shift nurses. Chronobiol Int. 2009; 26(7):1443-1461

47. Grundy A, Tranmer J, Richardson H, Graham $\mathrm{CH}$, Aronson KJ. The influence of light at night exposure on melatonin levels among Canadian rotating shift nurses. Cancer Epidemiol Biomarkers Prev. 2011;20(11):2404-2412.

48. Stevens RG, Hansen J, Costa G, et al. Considerations of circadian impact for defining "shift work" in cancer studies: IARC Working Group Report. Occup Environ Med. 2011;68(2):154-162.

49. Luckhaupt SE, Cohen MA, Li J, Calvert GM. Prevalence of obesity among U.S. workers and associations with occupational factors. Am J Prev Med. 2014;46(3):237-248.

50. Shields M, Connor Gorber S, Tremblay M. Estimations de l'obésité fondées sur des mesures autodéclarées et sur des mesures directes. Rapports sur la santé. 2008;19(2):61-76.
51. Statistique Canada. Base de données CANSIM : Tableau 105-0007: Indice de masse corporelle (IMC), selon le groupe d'âge et le sexe, population à domicile de 18 ans et plus excluant les femmes enceintes, Canada, provinces, territoires, régions sociosanitaires (limites de janvier 2000) et groupes de régions homologues, aux 2 ans [Internet]. Ottawa (Ont.) : Statistique Canada; [consulté le 11 févr. 2014]. En ligne à : http://www5.statcan.gc.ca /cansim

52. Williams C. L'équilibre travail-vie personnelle des travailleurs de quarts. L'emploi et le revenu en perspective. 2008; 75(5):5-18. 